\title{
Coupling the Neural and Physical Dynamics in Rhythmic Movements
}

\author{
Nicholas G. Hatsopoulos* \\ Division of Biology, CNS Program, MS 139-74, Caltech, Pasadena, CA 91125 USA
}

A pair of coupled oscillators simulating a central pattern generator (CPG) interacting with a pendular limb were numerically integrated. The CPG was represented as a van der Pol oscillator and the pendular limb was modeled as a linearized, hybrid spring-pendulum system. The CPG oscillator drove the pendular limb while the pendular limb modulated the frequency of the CPG. Three results were observed. First, sensory feedback influenced the oscillation frequency of the coupled system. The oscillation frequency was lower in the absence of sensory feedback. Moreover, if the muscle gain was decreased, thereby decreasing the oscillation amplitude of the pendular limb and indirectly lowering the effect of sensory feedback, the oscillation frequency decreased monotonically. This is consistent with experimental data (Williamson and Roberts 1986). Second, the CPG output usually led the angular displacement of the pendular limb by a phase of $90^{\circ}$ regardless of the length of the limb. Third, the frequency of the coupled system tuned itself to the resonant frequency of the pendular limb. Also, the frequency of the coupled system was highly resistant to changes in the endogenous frequency of the CPG. The results of these simulations support the view that motor behavior emerges from the interaction of the neural dynamics of the nervous system and the physical dynamics of the periphery.

\section{Introduction}

Traditional open-loop and closed-loop control schemes both assume the origins of rhythmic motor behavior reside in the nervous system in the form of central pattern generators (CPGs) (Delcomyn 1980). The spatial and temporal characteristics of the movement are dictated by central commands or reference signals, which are followed passively by the peripheral musculoskeletal system. In closed-loop schemes, afferent feedback acts to reduce any error between the reference signal and the actual position or velocity of the plant (Merton 1953; Stein 1982). Nevertheless,

*Correspondence should be sent to Nicholas G. Hatsopoulos, Department of Neuroscience, Box 1953, Brown University, Providence, RI 02912. 
the frequency and amplitude of the movement are centrally dictated by a series of set points.

Many open-loop and closed-loop schemes, however, suffer from inflexibility. They are unable to adapt to changing internal and external conditions that affect the musculoskeletal system. For example, the skeletal frame of a human can grow by over a factor of three from infancy to adulthood. Likewise, the mass of the body will increase by over one order of magnitude. Moreover, the body is exposed to transient loads when carrying objects or sustaining perturbations. In addition, the torque-generating capabilities of muscle can vary with time. Muscle can become more effective with exercise or less effective with injury or lack of use. All of these changes will alter the relationship between the neural signals emanating from the CPG and the resulting torques and movements generated about the joints.

The Russian physiologist Bernstein (1967) recognized this problem and proposed an approach to motor control that emphasized the exploitation of physical dynamics in movement generation (Schneider et al. 1989, 1990; Hoy and Zernicke 1985, 1986). This approach leads to the idea that stable movement patterns emerge from an interaction between the neural dynamics of the nervous system and the physical dynamics of the musculoskeletal system (Hatsopoulos 1992; Hatsopoulos et al. 1992). According to this approach, sensory afferents from the muscles, joints, and skin act to couple the physical dynamics to the neural dynamics just as the muscles couple the neural dynamics to the physical dynamics.

There are two important properties of motor rhythms that support this idea. First, the frequency of the motor rhythm can be modulated by changes in the strength of sensory feedback. In many systems, the frequency of a motor rhythm is higher with sensory feedback under intact conditions than it is without feedback (Wilson 1961; Grillner and Zangger 1979; Wallén and Williams 1984; Williamson and Roberts 1986; Mos and Roberts 1994). Wilson (1961) found that the wing beat frequency of the locust decreased gradually as more sensory nerves from the wings were cut. Wing beat frequency decreased by as much as a factor of two after complete deafferentation. Williamson and Roberts (1986) showed that the frequency of the dogfish swimming rhythm decreased as the extent of sensory feedback was indirectly decreased. This was accomplished by applying curare to the muscles controlling the movement.

The second and more important property is that the frequency of a motor rhythm depends on the inertial and gravitational properties of the periphery. In particular, the frequency of a motor rhythm will very often scale with or match the resonant frequency of the musculoskeletal system. ${ }^{1}$ The property of resonance tuning has been demonstrated in both observational field studies and in more formal experimental re-

\footnotetext{
${ }^{1}$ The resonant frequency of the musculoskeletal system can also depend on local, sensory feedback loops. Bässler (1983) showed that the closed-loop, femorotibial system of the stick insect possesses a resonant frequency between 1 and $3 \mathrm{~Hz}$. He postulated
} 
search (Kugler and Turvey 1987). Alexander and Jayes (1983) proposed an allometric model of walking that predicted a "pendular" scaling between the frequency of walking and body height: walking frequency under "normal" conditions should scale with the square root of the reciprocal of the body height. This prediction was borne out by observing the stepping frequency of a number of animals in the wild (Pennycuick 1975). More recently, Holt et al. (1990) asked subjects to walk with ankle weights of different masses and showed that they scale their preferred frequency with the resonant frequency of the leg.

Kugler and Turvey (1987) noted that both the stiffness of the joint and the physical properties of the limb (and any objects carried by the limb) will affect the resonant frequency of the periphery. They proposed a hybrid spring-pendulum model characterized by a forced, linear, secondorder differential equation with a sinusoidal forcing function whose amplitude and frequency are $T_{0}$ and $\omega$, respectively:

$$
I \ddot{\theta}+c \dot{\theta}+\left(m g l_{\mathrm{cm}}+k\right) \theta=T_{0} \sin \omega t
$$

where $I$ is the moment of inertia of the limb, $\theta$ is the angular displacement of the pendulum, $c$ is the damping coefficient, $m$ is the mass of the system, $g$ is the acceleration of gravity, $l_{\mathrm{cm}}$ is the distance of the center of mass from the axis of rotation, and $k$ is the stiffness of the joint. ${ }^{2}$ The angular resonant frequency ${ }^{3}$ of the system is

$$
\omega_{\mathrm{r}}=\sqrt{\left(m g l_{\mathrm{cm}}+k\right) / I}=\sqrt{\left(g / L_{\mathrm{eq}}\right)+(k / I)}
$$

where $L_{\mathrm{eq}}$ is the length of an equivalent simple pendulum whose mass is concentrated at one point. Kugler and Turvey hypothesized that the system tunes itself to a frequency that matches the resonant frequency of this hybrid model. Hatsopoulos and Warren (1995) have recently provided experimental support for this hypothesis.

Flight in insects and birds provides additional evidence for this hypothesis. Due to the size and orientation of the wings with respect to gravity, it is assumed that joint stiffness plays a larger role than does gravity, and, therefore, the second term in equation 1.2 will dominate. Sotavalta (1954) performed a number of experiments with moths and cockroaches in which the inertia of the wings was varied by either adding loads to the wings or clipping the wings. The wing beat frequency scaled with the inertia raised to a power ranging from -0.12 to -0.47 which is close to -0.5 predicted by the hypothesis. In an observational study, Greenewalt (1975) showed that the wing beat frequency of birds was proportional to the wing length raised to the power of -0.91 and higher.

that the stick insect takes advantage of the resonant frequency by tuning the frequency of its rocking behavior to that frequency.

${ }^{2}$ The pendulum is treated as a simple pendulum in these simulations so that $l_{\mathrm{cm}}=L$ where $L$ is the length of the pendulum. Also $I=m L^{2}$.

${ }^{3}$ Actually, the resonant frequency is shifted down slightly by the viscosity of the system: $w_{\mathrm{r}}=\sqrt{\left(m g l_{\mathrm{cm}}+k\right) / I-c^{2} / 4 I^{2}}$. 
If the length of the wing is much larger than the other two dimensions, the hypothesis would predict a scaling power of -1.0 .

I have shown previously how a spinal pattern generator model (Miller and Scott 1977) coupled to a pendular limb can exhibit the properties described above (Hatsopoulos et al. 1992). In this paper, I generalize these results by replacing the neural pattern generator with a more abstract, limit-cycle oscillator. I performed a set of simulations using a van der Pol oscillator coupled to a linearized hybrid spring-pendulum system. Since the nature of the pattern generator circuit for walking (in any system) is still poorly understood, it is important to demonstrate that these properties are a general consequence of the two-way coupling and not unique to one type of central pattern generator model.

\section{Method}

2.1 The Oscillators. The following equation describes the dynamics of the CPG modeled by a van der Pol oscillator, which yields a limit-cycle attractor in phase space:

$$
\ddot{y}+\epsilon\left(y^{2}-1\right) \dot{y}+\omega^{2} y=0
$$

where $y$ is the output of the CPG oscillator and is unitless, $\epsilon$ is a parameter that affects the shape of the limit-cycle, and $\omega$ is the angular frequency of the oscillator. The nonlinear, dissipative term is responsible for the existence of a limit cycle by acting either as a damper when $y$ is greater than 1 or as an excitor when $y$ is less than 1 . The dynamics of the limb segment were modeled by a linearized pendulum described by equation 1.1. The pendulum equation was linearized to make analysis simpler. If the oscillation amplitude is kept small $\left(<20^{\circ}\right)$, the linearization of the pendulum oscillator is appropriate (Seto 1964). The numerical simulations were performed with both the linearized and nonlinear versions of the pendulum equation. The results of the nonlinear version were qualitatively similar and so are not reported.

2.2 The Coupling Equations. The CPG oscillator drove the pendulum via a spring-like muscle whose resting or equilibrium length is proportional to the output of the CPG oscillator (Fel'dman 1966). ${ }^{4}$ The driving torque is, therefore, proportional to the difference between the position of the pendulum and the output of the oscillator:

$$
T=-k[\theta-(G / k) y]=-k \theta+G y
$$

\footnotetext{
${ }^{4}$ Actually, equation 2.2 represents the torque generated by an agonist-antagonist pair of muscles because a single muscle can generate torque only in one direction. Fel'dman (1966) demonstrated that under static conditions a fixed level of muscle activity defines a unique torque-angle curve with a certain equilibrium position.
} 
where $k$ is the stiffness of the muscle and $G$ is the muscle gain parameter whose units are in Newton-meters. If $k$ is zero, the neural oscillator acts like a pure torque driver. ${ }^{5}$ An alternate muscle model postulated by Hogan (1984) was also used in one set of simulations:

$$
T=-k y[\theta-(G / k)]=-k y \theta+G y
$$

In this model, the stiffness as well as the equilibrium position of the muscle are modulated by the output of the CPG oscillator. The results of this set of simulations are qualitatively similar to those using the first muscle model in relation to resonance tuning, and so are not reported in the Results section.

Positional feedback from the pendular limb acted on the CPG by modulating its frequency:

$$
\omega=\omega_{0}+B \theta
$$

where $\omega_{0}$ is the endogenous frequency of the CPG oscillator without proprioceptive feedback, $B$ is the feedback gain, and $\theta$ is the angular displacement of the pendular limb. Thus, the CPG model was a nonlinear, parametrically modulated oscillator. ${ }^{6}$ Two variations of this feedback equation were also used. First, the angular position of the pendulum was replaced with the absolute value of the angular position. In many ways, this represents a more realistic feedback model since muscle spindles act as rectifying position sensors. In this case, positional feedback comes from the agonist during one half of the cycle and from the antagonist muscle during the other half cycle. Second, positional feedback was replaced with torque feedback by using the full-wave rectified angular acceleration of the pendulum. The results using these two variations of the feedback equation were similar to the original as far as resonance tuning was concerned. That is, the frequency of the entrained system increased linearly with the resonant frequency of the pendulum for resonant frequencies ranging from 0.5 to about $1.1 \mathrm{~Hz}$. The results that are reported assume the feedback model represented in equation 2.4 unless otherwise noted.

There is indirect and direct experimental evidence for the general formulation of the feedback equation in which proprioceptive afferents act to modulate the endogenous frequency of the CPG. Some indirect evidence was reported above in which the removal of sensory feedback decreased the frequency of a motor rhythm (Wilson 1961; Grillner and Zangger 1979; Wallén and Williams 1984; Williamson and Roberts 1986; Mos and Roberts 1994). In addition, it has been demonstrated that tonic electrical stimulation from certain supraspinal areas in cat will induce fictive locomotion whose frequency is modulated by the stimulus strength (Shik et

\footnotetext{
${ }^{5} k$ was set to zero in all the simulations to be reported in this paper.

${ }^{6}$ The CPG model is basically a hybrid van der Pol and Mathieu oscillator (Jordan and Smith 1989).
} 
Table 1: Parameter Values Used in Simulations

\begin{tabular}{cc}
\hline$m(\mathrm{~kg})$ & 10 \\
$L(\mathrm{~m})$ & $0.1-10.0$ \\
$c(\mathrm{~N}-\mathrm{m}-\mathrm{sec} / \mathrm{rad})$ & $0.5-1.0$ \\
$\epsilon$ & 0.5 \\
$G(\mathrm{~N}-\mathrm{m})$ & $0.25-1.6$ \\
$B\left(\mathrm{sec}^{-1}\right)$ & $0-50$ \\
$\omega_{0}(\mathrm{rad} / \mathrm{sec})$ & $0-5$ \\
\hline
\end{tabular}

al. 1966). This suggests that tonic synaptic input to the pattern generator network can affect its output frequency. Therefore, if proprioceptive afferents have symaptic influence on the pattern generator network, they could, in principle, affect the CPG frequency. More direct evidence includes the finding that tonic electrical stimulation of sensory nerves in the thoracic ganglia of locusts will increase the wing beat frequency (Wilson 1964).

The simulations involved the numerical integration of the coupled set of differential equations with a fourth-order Runge-Kutta integrator whose time step was fixed at either 2.5 or $5 \mathrm{msec}$. Table 1 shows the range of parameter values used in the simulations.

\section{Results}

3.1 Frequency vs. Amplitude. I conducted a set of simulations to investigate the relationship between oscillation frequency and amplitude in the pendular limb. The amplitude of oscillations was modulated by changing the muscle gain, $G$, in equation 2.2. As expected, the oscillation amplitude decreased as the muscle gain was decreased. More surprisingly, the oscillation period increased as the amplitude decreased. The period and amplitude of the pendular limb are plotted as a function of cycle number in Figure 1A. These step-wise changes in muscle gain were performed to simulate the effects of intravenous injection of curare into the muscles of the dogfish mediating swimming (Williamson and Roberts 1986) (Fig. 1B). As the curare takes effect, the period of the oscillating body increases as the amplitude decreases. After about $80 \mathrm{sec}$ from the time of injection, the period and amplitude return to their normal values.

3.2 Phase. A striking feature of the simulations is the relative phase between the torque generated by the CPG oscillator and the angular displacement of the pendular limb (Fig. 2). The endogenous frequency of the CPG oscillator without feedback, $\omega_{0}$, is set at $1 \mathrm{rad} / \mathrm{sec}$ or $0.16 \mathrm{~Hz}$. The length of the pendulum is set at one of four values: $0.1,0.2,0.4$, and 
A Simulation

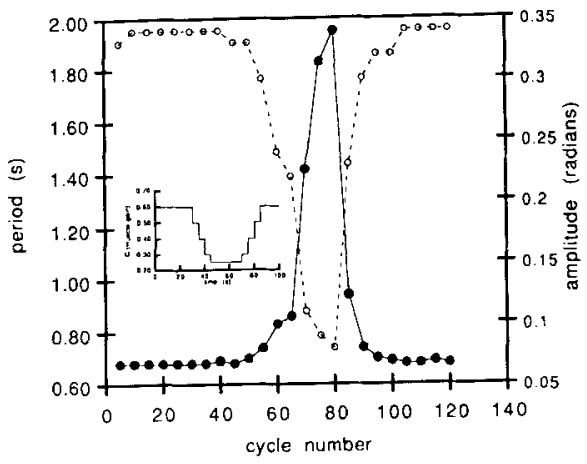

B Experiment

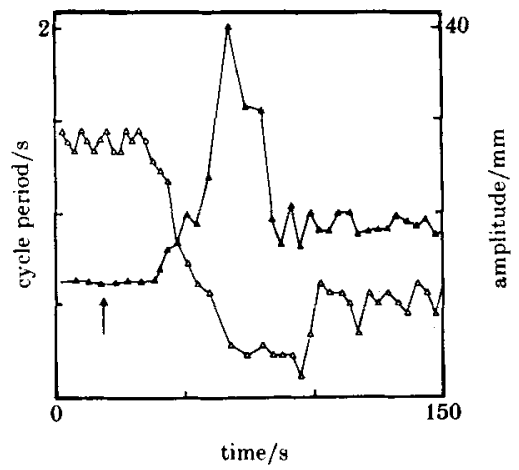

Figure 1: (A) The amplitude (open circles) and period (solid circles) of the pendular limb versus cycle number as the muscle gain, $G$, is varied. Inset: muscle gain, $G$, as a function of time. (B) The amplitude (open triangles) and period (solid triangles) of the dogfish swimming rhythm versus time as the level of curare in the muscle varied (copied from Williamson and Roberts 1986). Intravenous injection of curare is applied at the arrow.

$0.8 \mathrm{~m}$, which corresponds to a resonant frequency of about 1.58, 1.11, 0.79 , and $0.56 \mathrm{~Hz}$, respectively. With feedback, the torque and, therefore, the CPG output (the torque is in-phase with the the CPG output; the two 
A WITH FEEDBACK
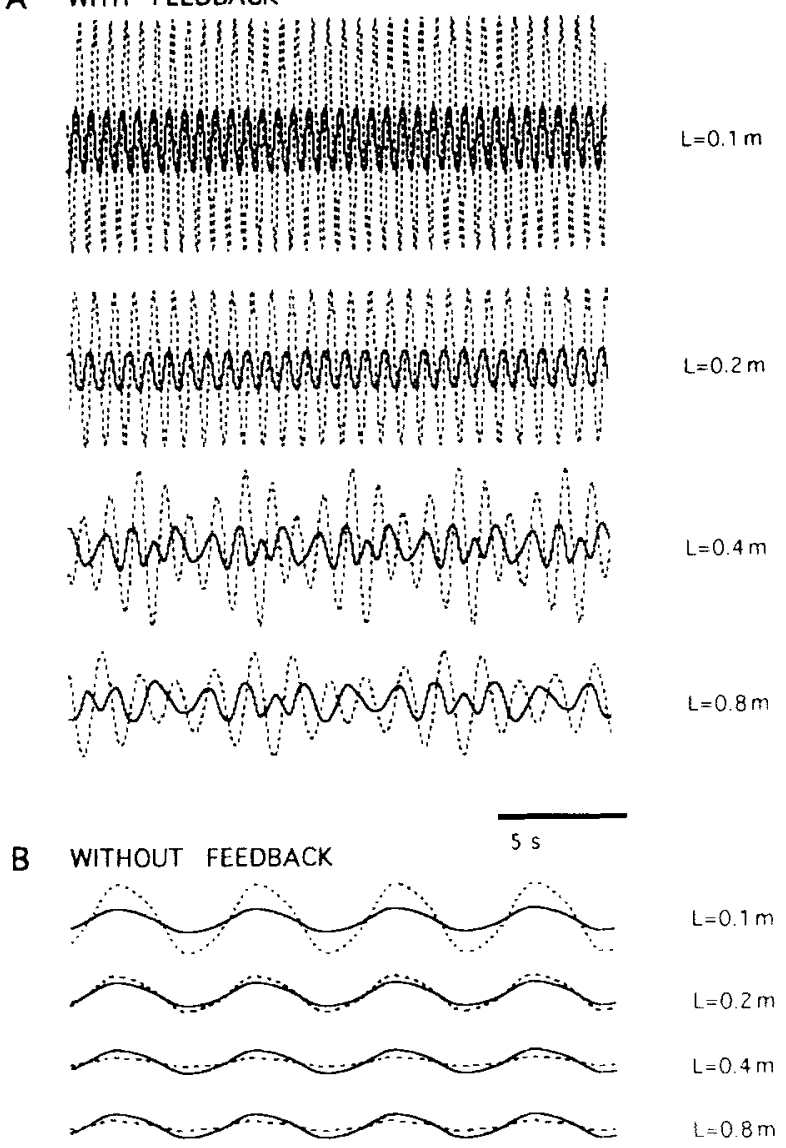

Figure 2: The torque generated by the CPG (solid lines) and angular displacement of the pendular limb (dotted lines) as a function of time for four different pendulum lengths. (A) With feedback. Notice that the torque generally leads the angular displacement of the pendulum by $90^{\circ}$. For $L=0.4$ and $L=0.8$, the phase lead varies from cycle to cycle between 90 and over $180^{\circ}$. Also, notice how the frequency and amplitude of the pendulum's oscillations decrease as the length of the pendulum increases. The feedback signal used in these simulations was the full-wave rectified angular position of the pendulum. (B) Without feedback. The torque is in-phase with the angular displacement of the pendulum. Only the amplitude of the pendulum's oscillations decreases as the length of the pendulum increases. The frequency is fixed and determined by the endogenous frequency of the CPG oscillator: $0.16 \mathrm{~Hz}$. 
differ only by the factor, $G$ ) generally lead the pendulum's displacement by a phase of approximately $90^{\circ}$ (Fig. 2A). The significance of this phase lead is mentioned in the Discussion. For a pendulum length of 0.4 and $0.8 \mathrm{~m}$, the relative phase between the two oscillators changes from cycle to cycle. The phase lead of the CPG output varies between 90 and over $180^{\circ}$. Also, the amplitude and frequency of the two oscillators vary from cycle to cycle. The average frequency and amplitude of the pendulum's oscillations decrease as the length of the pendulum increases.

In the absence of feedback, the two oscillators are nearly in-phase for all pendulum lengths (Fig. 2B). Notice that the amplitude of the pendulum's oscillations decreases as the pendulum length increases. In contrast, the frequency remains fixed at $0.16 \mathrm{~Hz}$ because the pendulum is acting like a passively driven system.

3.3 Resonance Tuning. I performed another set of simulations to investigate the ability of the coupled system of oscillators to tune its frequency to the resonant frequency of the pendular limb. The length of the pendulum was decreased so that its resonant frequency increased by a factor of 3 . The average frequency at which the coupled system (i.e., both the CPG and the pendular limb) settled is plotted as a function of the resonant frequency of the pendular limb for three different values of the muscle gain, $G$ (Fig. 3A, with feedback). Notice that the average frequency of the system scaled with or nearly matched the resonant frequency of the pendulum. Although resonance matching required a particular set of parameter values, resonance scaling was a robust phenomenon as long as the resonant frequency of the pendular limb was higher than the endogenous frequency, $\omega_{0}$, of the CPG oscillator. If the resonant frequency of the limb approached (or fell below) the endogenous frequency of the CPG oscillator, two phenomena occurred. First, the system entrained to the endogenous fequency of the CPG oscillator. For example, if the endogenous frequency was set to $1.6 \mathrm{~Hz}\left(\omega_{0}=10.0\right)$, the pendular limb settled at that frequency despite variations in the resonant frequency when the resonant frequency fell below the endogenous frequency (Fig. 3B, hollow circles). Second, the system often entered a regime of subharmonic entrainment in which the pendular limb swung at its resonant frequency while the CPG oscillated at an integer multiple of the pendular limb's frequency. Resonance tuning also broke down at resonant frequencies much larger than the endogenous frequency of the CPG (Fig. 3B, all three simulations). Resonance scaling was observed for muscle gains that varied by as much as one order of magnitude and for feedback gains ranging from under 5 up to 50 .

When feedback was removed $(B=0)$, the pendular limb acted like a passively driven system whose frequency was dictated by the CPG's frequency: the limb frequency remained constant as the resonant fre- 

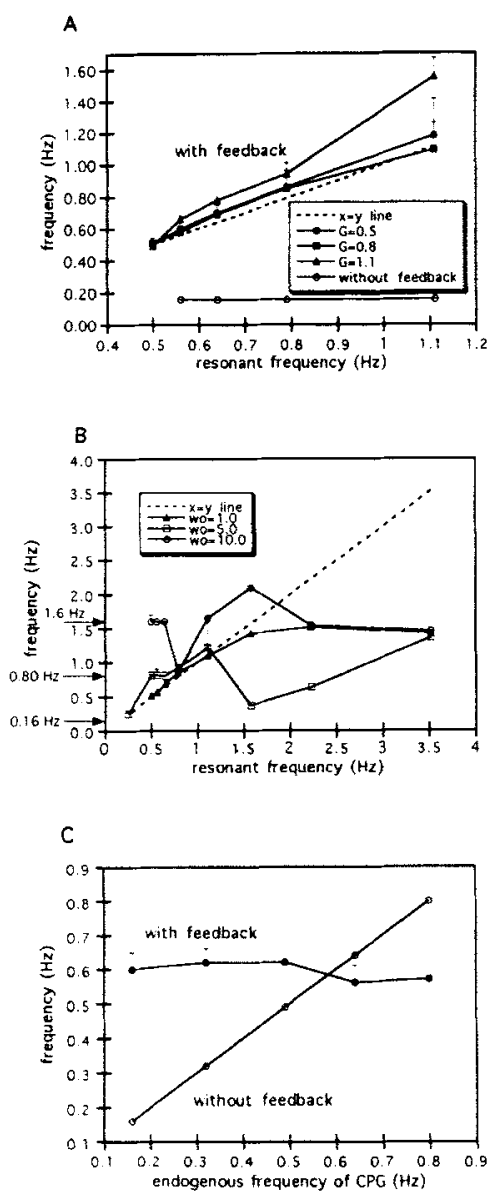

Figure 3: (A) The frequency of the coupled system as a function of resonant frequency of the pendular limb without feedback (open circles) and with feedback for three different muscle gain values (solid circles, $G=0.5$; solid squares, $G=0.8$; solid triangles, $G=1.1$ ). The endogenous frequency is held at $0.16 \mathrm{~Hz}$. (B) The frequency of the pendular limb as a function of resonant frequency for three different endogenous frequenices. Notice how the pendular limb entrains to the endogenous frequency when the resonant frequency is comparable to or smaller than the endogenous frequencies $\omega_{\omega}^{\prime}=5.0(0.8 \mathrm{~Hz})$ and $\omega_{0}=10.0$ (1.6 Hz)]. (C) The frequency of the coupled system as a function of the CPG's endogenous frequency without feedback (open circles) and with feedback (solid circles). The resonant frequency of the pendular limb is held at $0.56 \mathrm{~Hz}$. The error bars represent standard deviations of the frequency due to variability from cycle to cycle. 
quency increased (Fig. 3A, without feedback). ${ }^{7}$ It is important to note that resonance tuning required that sensory feedback modulate the endogenous frequency of the CPG oscillator. When the feedback equation was modified such that proprioception from the pendulum acted on the CPG oscillator as a driving torque as opposed to a frequency modulator, the frequency of the coupled system remained fixed and did not vary with the resonant frequency of the pendulum. That is, the two oscillators always entrained to the endogenous frequency of the CPG oscillator.

I performed a complementary set of simulations in which the resonant frequency of the pendular limb was held fixed while the endogenous frequency, $\omega_{0}$, of the CPG oscillator was increased (Fig. 3C). The frequency of the coupled system remained relatively constant near the resonant frequency as the endogenous frequency was increased. On the other hand, the frequency of the system tracked the endogenous frequency of the CPG oscillator when sensory feedback was removed.

3.4 Frequency Control. This model raises the question of how the nervous system can influence the oscillation frequency of the pendular limb if its frequency is so resistant to changes in the CPG's endogenous frequency. There are at least three methods by which the CPG oscillator might control the frequency of the pendular limb. First, I have shown how the muscle gain, $G$, can affect both the frequency and amplitude of the motor rhythm. Second, modulating the stiffness of the joint $(k$ in equation 2.2) will change the frequency of the system. Joint stiffness can be changed either by changing the stiffnesses of individual muscles or by co-contracting agonist-antagonist pairs of muscles. In either case, an increase in joint stiffness will increase the resonant frequency of the hybrid, spring-pendulum system and, therefore, increase the frequency of the pendular limb. Simulations have demonstrated the feasibility of such a method. Third, if sensory feedback can be gated by the nervous system, the pendular limb will become a passively driven system whose frequency will track the endogenous frequency of the CPG (see Fig. 3C, without feedback).

\section{Discussion}

I have presented the results of a number of simulations to support the idea that rhythmic movements emerge from the interaction of the neural dynamics of the nervous system and the physical dynamics of the periphery. In particular, I have shown that modeling feedback as a parametric coupling of the CPG's frequency generates the amplitude-frequency relationship observed experimentally (Williamson and Roberts 1986).

\footnotetext{
${ }^{7}$ On occasion, subharmonic entrainment was observed even without sensory feedback.
} 
I have demonstrated that the relative phase between the CPG output and the pendulum's angular displacement with sensory feedback remains generally invariant at $90^{\circ}$ despite variations in the system's frequency induced by changes in the pendulum length (see Fig. 2A). The phase transfer function of a second-order system such as a linearized pendulum varies from 0 to $180^{\circ}$ as the driving frequency increases. In fact, the phase transfer function is particularly sensitive to changes in driving frequency near $90^{\circ}$. So how does the CPG output maintain a $90^{\circ}$ phase lead relative to the pendulum's displacement? Proprioceptive feedback allows the central pattern generator to tune its frequency to the resonant frequency of the periphery (see Fig. 3A). The phase transfer function of a second-order system attains a value of $90^{\circ}$ at its resonant frequency. On the other hand, the two oscillators are in-phase in the absence of sensory feedback because the endogenous frequency of the CPG oscillator without feedback is much lower than the resonant frequency of the pendulum $\left(\omega_{0} / 2 \pi=0.16 \mathrm{~Hz}\right)$ in accord with the phase transfer function.

Resonance tuning seems to be a general feature of a limit-cycle oscillator coupled to a pendulum with this form of parametric feedback. If the viscous term in the van der Pol oscillator is modified by replacing $y^{2}$ with $y^{4}$, the system remains a limit-cycle oscillator, and resonance tuning is observed. I have also shown the existence of resonance tuning when the formal limit-cycle oscillator is replaced with a sixth-order, neural oscillator with limit-cycle stability (Hatsopoulos et al. 1992).

A simplistic way to understand how resonance tuning arises is to consider the interaction of the frequency response of the pendulum with the feedback equation. The frequency response of a second-order system relates its oscillation amplitude to the driving frequency and has a peak at its resonant frequency. The feedback equation determines indirectly how the frequency of the CPG (i.e., the driving frequency) will depend on the oscillation amplitude of the pendulum because the equation relates the endogenous frequency of the CPG oscillator to the instantaneous displacement of the pendulum. In the case of the modified feedback equation involving the rectified displacement, the larger the amplitude of the pendulum, the larger the temporal average of the pendulum's rectified displacement and, therefore, the CPG's endogenous frequency. Therefore, the frequency and amplitude at which the pendulum equilibrates are determined by the intersection of its frequency response with the curve relating the frequency of the CPG with the amplitude of the pendulum. As the resonant frequency of the pendulum increases, the frequency response curve shifts horizontally along the frequency axis. Therefore, the intersection point will also shift to a higher frequency. This is similar to the economic concept that the price of a product depends on the intersection of the supply and demand curves.

Resonance tuning is an important property for at least two reasons. First, driving a physical system at its resonant frequency requires a min- 
imum driving torque for a fixed movement amplitude. This implies that the metabolic costs of driving a limb at its resonant frequency are minimized. This can be viewed in another way. Since the torque leads the displacement of the pendular limb by $90^{\circ}$, the time-integral of the product of the external torque and angular velocity over a period of the rhythm (i.e., the work done by the muscles on the pendulum) is maximized. Second, resonance tuning stabilizes the movement by making the movement frequency resistant to fluctuations in the endogenous frequency of the CPG.

This model of rhythmic motor control makes a number of predictions that can be tested experimentally. First, under most conditions ${ }^{8}$ the net torque should lead the displacement of the limb by $90^{\circ}$. Hatsopoulos and Warren (1995) provided evidence for this in human arm movements about the elbow joint. Second, feedback should act to stabilize the motor rhythm's frequency. Some evidence for this comes from work on motor rhythms in the eel (Mos and Roberts 1994). Third, it suggests that movement frequency should increase with joint stiffness (Latash 1992). Finally, movement frequencies away from resonance could also involve the gating or blocking of sensory feedback.

\section{Acknowledgments}

This research was partially funded by a grant from the Office of Naval Research to Gilles Laurent. I thank Micah Siegel and William H. Warren for their very helpful comments on this manuscript.

\section{References}

Alexander, R. M., and Jayes, A. S. 1983. A dynamic similarity hypothesis for the gaits of quadrupedal mammals. I. Zool. London 201, 135-152.

Bässler, U. 1983. Neural Basis of Elementary Behavior in Stick Insects. SpringerVerlag, Berlin.

Bernstein, N. A. 1967. The Coordination and Regulation of Movements. Pergamon Press, London.

Delcomyn, F. 1980. Neural basis of rhythmic behavior in animals. Science 210, 492-498.

Fel'dman, A. G. 1966. Functional tuning of the nervous system during control of movement or maintenance of a steady posture. II. Controllable parameters of the muscle. Biophysics 11, 565-578.

Greenewalt, C. H. 1975. The flight of birds. Transact. Am. Philos. Soc. 65, 21-23.

Grillner, S., and Zangger, P. 1979. On the central generation of locomotion in the low spinal cat. Exp. Brain Res. 34, 241-261.

\footnotetext{
${ }^{8}$ There may be particular circumstances in which sensory feedback is gated so that rhythmic movement can occur away from resonance.
} 
Hatsopoulos, N. G. 1992. The coupling of neural and physical dynamics in motor control. Unpublished doctoral dissertation, Brown University, Providence.

Hatsopoulos, N. G., Warren, W. H., and Sanes, I. N. 1992. A neural pattern generator that tunes into the physical dynamics of the limb system. Int. Joint Conf. Neural Neticorks '92, I, 104-109. Baltimore, MD.

Hatsopoulos, N. G., and Warren, W. H. 1995. Resonance tuning in rhythmic arm movements. J. Motor Beha'', in press.

Hogan, N. 1984. Adaptive control of mechanical impedance by coactivation of antagonist muscles. IEEE Transact. Automatic Control 29, 681-690.

Holt, K. G., Hamill, J, and Andres, R. O. 1990. The force-driven harmonic oscillator as a model for human locomotion. Human Movement Sci. 9, 55-68.

Hoy, M. G., and Zernicke, R. F. 1985. Modulation of limb dynamics in the swing phase of locomotion. J. Biomech. 18, 49-60.

Hoy, M. G., and Zernicke, R. F. (1986). The role of intersegmental dynamics during rapid limb oscillations. J. Biomech. 19, 867-877.

Jordan, D. W., and Smith, P. 1989. Nonlinear Ordinary Differential Equations. Clarendon Press, Oxford.

Kugler, P. N., and Turvey, M. T. 1987. Information, Natural Law, and the SelfAssembly of Rhythmic Moc'me'nt. Lawrence Erlbaum, Hillsdale, NJ.

Latash, M. L. 1992. Virtual trajectories, joint stiffness, and changes in the limb natural frequency during single-joint oscillatory movements. Neturoscience 49, 209-220.

Merton, P. A. 1953. Speculations on the servo-control of movement. In CIBA Fotndation Symposium, The Spinal Chord, G. E. W. Wolstenholme (ed.), pp. 247255. Churchill, London.

Miller, S., and Scott, P. D. 1977. The spinal locomotor generator. Exp. Brain Res. 30, 387-403.

Mos, W., and Roberts, B. L. 1994. The entrainment of rhythmically discharging reticulospinal neurons of the eel by sensory nerve stimulation. I. Comp. Physiol. A 174, 391-397.

Pennycuick, C. J. 1975. On the running of the gnu (Connochaetes taurinus) and other animals. J. Exp. Biol. 63, 775-799.

Schneider, K., Zernicke, R. F., Schmidt, R. A., and Hart, T. J. 1989. Changes in limb dynamics during the practice of rapid arm movements. J. Biomech. 22, 805-817.

Schneider, K., Zernicke, R. F., Ulrich, B. D., Jensen, J., and Thelen, E. 1990. Understanding movement control in infants through the analysis of limb intersegmental dynamics. J. Motor Behav. 22, 493-520.

Seto, W. W. 1964. Theory and Problems of Mechanical Vibrations. Schaum, New York.

Shik, M. L., Severin, F. V., and Orlovsky, G. N. 1966. Control of walking and running by means of electrical stimulation of the mid-brain. Biophysics 11, $756-765$.

Sotavalta, O. 1954. The effect of wing inertia on the wing stroke frequency of moths, dragonflies, and cockroach. Ann. Ent. Fenn. 20, 93-100. 
Stein, R. B. 1982. What muscle variable(s) does the nervous system control in limb movements? Behav. Brain Sci. 5, 535-577.

Wallén, P., and Williams, T. L. 1984. Fictive locomotion in the lamprey spinal cord in vitro compared with swimming in the intact and spinal animal. J. Physiol. London 347, 225-239.

Williamson, R. M., and Roberts, B. L. 1986. Sensory and motor interactions during movement in the spinal dogfish. Proc. Royal Soc. London Ser. B 227, 103-119.

Wilson, D. M. 1961. The central nervous control of flight in a locust. J. Exp. Biology 38, 471-490.

Wilson, D. M. 1964. The origin of the flight-motor command in grasshoppers. In Neuronal Theory and Modeling, R. F. Reiss (ed.), pp. 331-345. Stanford University Press, Stanford, CA.

Received July 22, 1994; accepted August 14, 1995. 